JOURNAL OF

SYNCHROTRON

RADIATION

ISSN 1600-5775

Received 5 December 2015

Accepted 19 February 2016

Edited by S. Svensson, Uppsala University, Sweden

Keywords: hard X-ray photoemission; linear dichroism; strongly correlated electron systems; phase retarder; micro-focused X-rays; sample monitoring system; low-temperature double-axis manipulator.

\section{Polarized hard X-ray photoemission system with micro-positioning technique for probing ground- state symmetry of strongly correlated materials}

\author{
Hidenori Fujiwara, ${ }^{\mathrm{a}, \mathrm{b} *}$ Sho Naimen, ${ }^{\mathrm{a}}$ Atsushi Higashiya, ${ }^{\mathrm{b}, \mathrm{c}}$ Yuina Kanai, ${ }^{\mathrm{a}, \mathrm{b}}$ \\ Hiroshi Yomosa, ${ }^{\mathrm{a}, \mathrm{b}}$ Kohei Yamagami, ${ }^{\mathrm{a}, \mathrm{b}}$ Takayuki Kiss, ${ }^{\text {a,b }}$ Toshiharu Kadono, \\ Shin Imada, ${ }^{\text {b,d }}$ Atsushi Yamasaki, ${ }^{\text {bee }}$ Kouichi Takase, ${ }^{\text {, }}$ Shintaro Otsuka, ${ }^{\text {g }}$ \\ Tomohiro Shimizu, ${ }^{\text {g }}$ Shoso Shingubara, ${ }^{\text {g }}$ Shigemasa Suga, ${ }^{\text {,h }}$ Makina Yabashi, ${ }^{\text {b }}$ \\ Kenji Tamasaku, ${ }^{b}$ Tetsuya Ishikawa $^{b}$ and Akira Sekiyama ${ }^{a, b}$ \\ a'Division of Materials Physics, Graduate School of Engineering Science, Osaka University, Toyonaka, Osaka 560-8531,
Japan, ${ }^{\mathbf{b}}$ SPring-8/RIKEN, Sayo, Hyogo 679-5148, Japan, ${ }^{\mathbf{c}}$ Faculty of Science and Engineering, Setsunan University,
Neyagawa, Osaka 572-8508, Japan, ${ }^{\mathbf{d}}$ Department of Physical Science, Ritsumeikan University, Kusatsu, Shiga 525-8577,
Japan, ${ }^{\mathbf{e}}$ Faculty of Science and Engineering, Konan University, Kobe 658-8501, Japan, ' ${ }^{\mathbf{D}}$ Department of Physics, College
of Science and Technology, Nihon University, Chiyoda, Tokyo 101-0062, Japan, ${ }^{\mathbf{g}}$ Graduate School of Science and
Technology, Kansai University, Suita, Osaka 564-8680, Japan, and ${ }^{\mathbf{h}}$ Institute of Scientific and Industrial Research,
Osaka University, Ibaraki, Osaka 567-0047, Japan. ${ }^{*}$ Correspondence e-mail: fujiwara@mp.es.osaka-u.ac.jp
}

An angle-resolved linearly polarized hard X-ray photoemission spectroscopy (HAXPES) system has been developed to study the ground-state symmetry of strongly correlated materials. The linear polarization of the incoming X-ray beam is switched by a transmission-type phase retarder composed of two diamond (100) crystals. The best value of the degree of linear polarization was found to be -0.96 , containing a vertical polarization component of $98 \%$. A newly developed low-temperature two-axis manipulator enables easy polar and azimuthal rotations to select the detection direction of photoelectrons. The lowest temperature achieved was $9 \mathrm{~K}$, offering the chance to access the ground state even for strongly correlated electron systems in cubic symmetry. A co-axial sample monitoring system with long-working-distance microscope enables the same region on the sample surface to be measured before and after rotation. Combining this sample monitoring system with a micro-focused X-ray beam by means of an ellipsoidal Kirkpatrick-Baez mirror $(25 \mu \mathrm{m} \times 25 \mu \mathrm{m}$ FWHM), polarized valence-band HAXPES has been performed on $\mathrm{NiO}$ for voltage application as resistive random access memory to demonstrate the micropositioning technique and polarization switching.

\section{Introduction}

Strongly correlated electron systems show a variety of anomalous phenomena such as high- $T_{\mathrm{c}}$ superconductivity and metal-insulator transitions, attracting much attention in solid state physics. In the case of transition-metal $3 d$-electron systems, the ground-state symmetry can easily be determined due to the crystal-field splitting of eV order. However, the situation is different in the case of rare-earth $4 f$-electron systems in which the crystal-field splitting is usually of $\mathrm{meV}$ order. Therefore, the determination of the ground-state symmetry for the $4 f$-electron systems is still a non-trivial issue, although it is crucial for discussing the origin of intriguing phenomena such as quantum criticality, heavy-Fermion superconductivity and non-Fermi liquid behavior. For this purpose, the linear polarization dependence of soft X-ray absorption spectra (XAS) has been well established as a local probe to detect the symmetry of the electronic structure due 
to excitonic excitation forming a core hole in the final states with dipole selection rules. This technique is powerful for $\mathrm{Ce}$ compounds with tetragonal or orthorhombic crystal structures (Hansmann et al., 2008; Willers et al., 2009, 2010, 2012, 2015; Strigari et al., 2012, 2013), but does not work for a high symmetric cubic system because the selection rule is only sensitive to cases where the electric field vector of the excitation photon is parallel or perpendicular to the anisotropic crystal axis. This fundamental limitation is, however, overcome by the recent development of angle-resolved 'linealy polarized' hard X-ray photoemission spectroscopy (HAXPES) (Mori et al., 2014), in which dipole selection rules work equally well as polarized XAS. Moreover, we have another controllable probing parameter, i.e. the photoelectron-detection direction, enabling the ground-state symmetry to be probed even for cubic systems (Kanai et al., 2015). This type of polarized HAXPES is a brand new spectroscopy for discussing the ground-state symmetry in contrast to the so far reported polarized HAXPES technique which is mainly used to study the electronic structures utilizing the angular-momentum dependence of photoelectron angular distributions due to the atomic photo-ionization cross section (Sekiyama et al., 2010, 2013; Nakatsu et al., 2011; Weinen et al., 2015) or magnetic circular dichroism (Ueda et al., 2008; Ouardi et al., 2011; Kozina et al., 2011).

In this paper we report on the performance of a HAXPES system with polarization switching potential at SPring-8 BL19LXU. Double phase retarders can switch the polarization of the incoming photon from horizontal to vertical polarization with the degree of linear polarization $P_{\mathrm{L}}=-0.96$. A twoaxis manipulator with lowest available temperature of $9 \mathrm{~K}$ has been newly developed to easily select the photoelectrondetection direction in the ground state of strongly correlated electron systems. Moreover, a sample monitoring system with co-axial long-working-distance microscope enables one to keep measuring the same sample region after rotation. Combined with a micro-focused X-ray beam of size $25 \mu \mathrm{m} \times$ $25 \mu \mathrm{m}$ (FWHM), we further demonstrate the linear polarization dependence of the valence-band HAXPES spectra for voltage-applied $\mathrm{NiO}$ surrounded by $200 \mu \mathrm{m} \times 200 \mu \mathrm{m} \mathrm{Pt}$ markers to test the polarization switching and the micropositioning technique.

\section{HAXPES system with linear polarization switching}

\subsection{Beamline setup and double-crystal phase retarder}

Polarization-dependent HAXPES was performed at BL19LXU at SPring-8 using an MBS A1-HE hemispherical photoelectron spectrometer which is inclined at $60^{\circ}$ from the incident X-ray beam. Fig. 1 shows a schematic diagram of the typical setting of the beamline optics for polarized HAXPES. Linearly polarized light is delivered from an in-vacuum $27 \mathrm{~m}$ long 780 period undulator (Kitamura et al., 2001; Yabashi et al., 2001a,b). The photon energy is set to $\sim 7.9 \mathrm{keV}$ by the $\mathrm{Si}(111)$ double-crystal monochrometer and further monochromated by the $\mathrm{Si}(620)$ channel-cut crystal. The transmis-

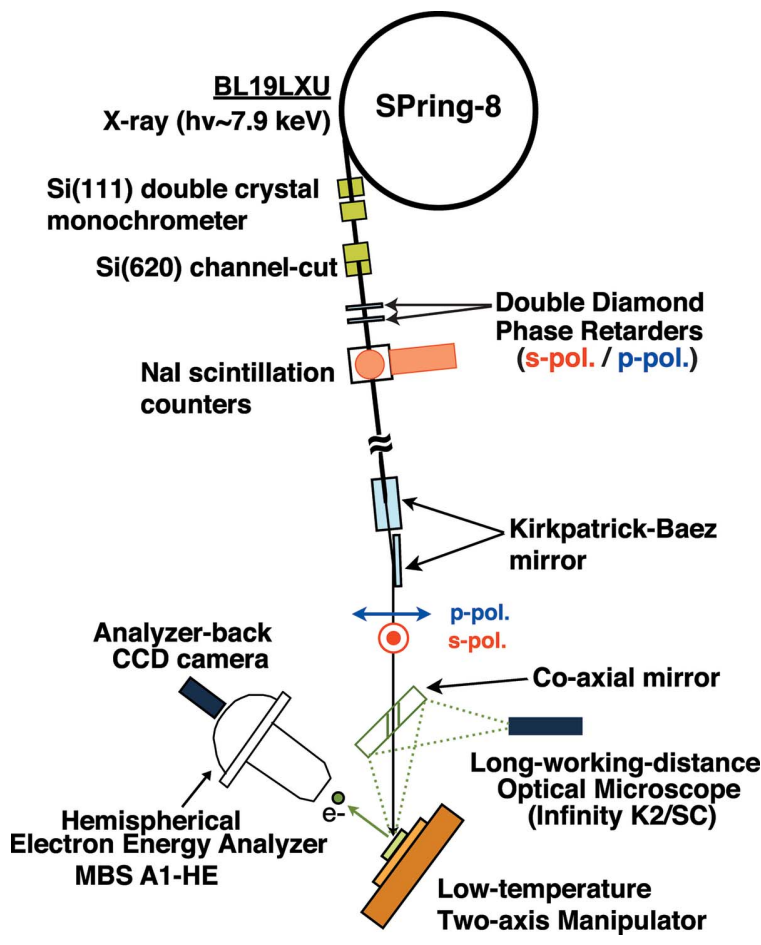

Figure 1

Overview of the experimental geometry (top view) for polarized HAXPES at BL19LXU in SPring-8.

sion-type phase retarder made of two single-crystalline (100) diamonds is placed downstream of the channel-cut crystal to switch the polarization of the X-ray beam (Hirano et al., 1991, 1993; Hirano \& Maruyama, 1997; Giles et al., 1994). To compensate for the phase-shift inhomogeneity due to the angular divergence of the incoming X-ray beam, we use the double-phase-plate configuration (Okitsu et al., 2001), giving the (220) Bragg reflection with Laue geometry, whose scattering plane is inclined at $45^{\circ}$ to the electric field vector of the incoming X-ray beam. The original horizontally polarized photons are transformed into circular polarization by the first diamond plate (Fig. 2a), and then the offset angle of the second plate is scanned as shown in Fig. 2(b) to give a further phase shift for converting to vertical polarization (Scagnoli et $a l ., 2009)$. The degree of linear polarization is defined as

$$
P_{\mathrm{L}}=\frac{I_{\mathrm{h}}-I_{\mathrm{v}}}{I_{\mathrm{h}}+I_{\mathrm{v}}}
$$

where $I_{\mathrm{h}}$ and $I_{\mathrm{v}}$ are intensities of the horizontal and vertical polarized photons, respectively. $I_{\mathrm{h}}$ and $I_{\mathrm{v}}$ are evaluated using scattering photon intensities from polyimide film detected with $\mathrm{NaI}$ scintillation counters located downstream of the phase retarder. In our setup, the best $P_{\mathrm{L}}$ value is evaluated as -0.96 as shown in Fig. 2(b). This gives the fraction of vertical components of $98 \%$, having sufficient quality to enable discussion of the linear polarization dependence of the photoemission spectra. Note that the electric field vector of the horizontal (vertical) polarized photons is parallel (perpendicular) to the photoelectron scattering plane, and thus defined as $p$-polarization (s-polarization). The thickness 

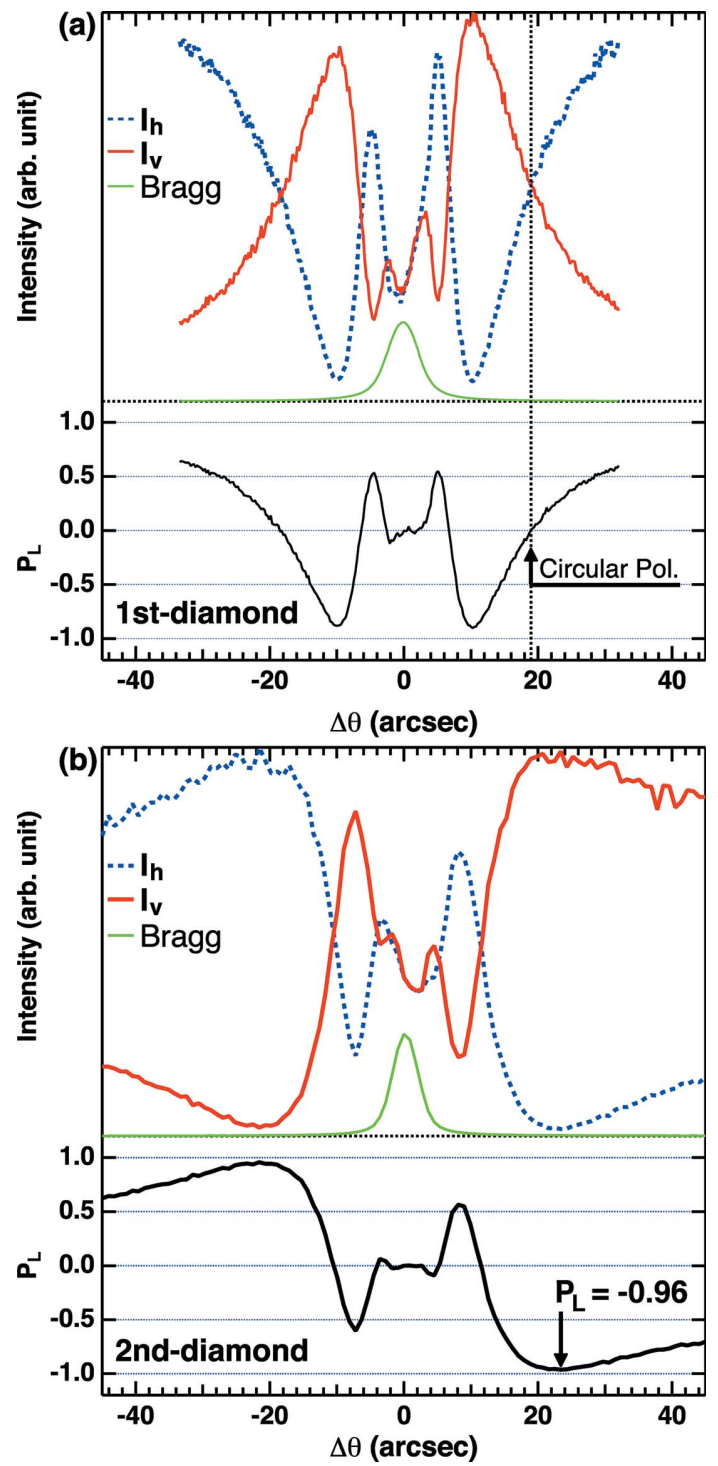

Figure 2

Offset angle dependence of the photon intensities relative to the diamond (220) Bragg reflections for the first $(a)$ and second $(b)$ diamond. The evaluated degree of linear polarization $P_{\mathrm{L}}$ is also plotted at the bottom of $(a)$ and $(b)$. of each diamond plate is $0.25 \mathrm{~mm}$, and the transmittance of the $\mathrm{X}$-ray beam after the double-crystal phase retarder is $\sim 50 \%$, which gives a much better throughput for the vertically polarized photons than that for a $0.7 \mathrm{~mm}$-thick single phaseretarder setup with transmittance of $\sim 35 \%$ (Sekiyama et al., 2010, 2013; Nakatsu et al., 2011). Afterwards the X-ray beam is focused onto the sample within $25 \mu \mathrm{m} \times 25 \mu \mathrm{m}$ (FWHM) using an ellipsoidal Kirkpatrick-Baez mirror as shown in Fig. 1.

\subsection{Low-temperature two-axis manipulator}

We have developed a two-axis manipulator for polarized HAXPES to easily optimize the detection direction of photoelectrons. Fig. 3(a) shows schematic drawings of the manipulator. The body of the manipulator is made of oxygenfree copper with gold plating. The rotation feedthrough provides polar rotation $(\theta)$, and a rotation stage made of Be$\mathrm{Cu}$ on top of the manipulator gives the azimuthal rotation $(\varphi)$ over a $90^{\circ}$ range. The bottom of the rotation stage is bowlshaped to gain thermal contact, and is tightly fixed by a nonmagnetic screw from the back. This screw is loosened during the azimuthal rotation, but four screws support the rotation stage to prevent it from falling during rotation, as shown in Fig. 3(b). By minimizing the volume of the manipulator, we have achieved a lowest temperature of $9 \mathrm{~K}$ using a closed-cycle He refrigerator, enabling cooling to $9 \mathrm{~K}$ in $1.5 \mathrm{~h}$ from $300 \mathrm{~K}$. Although the azimuth stage loses thermal contact during rotation, one can immediately cool down the sample after retightening the rotation stage screw. The sample is mounted on the rotation stage by clamping between two aluminium fingers with a screw to hold the sample using a hexagonal wrench from the back, and the same wrench can be used to manipulate the rotation stage. To guide the rotation angle, there are seven indication marks every $15^{\circ}$.

The rotation angle is fixed more precisely by monitoring the sample with a charge-coupled device (CCD) camera, which is mounted on the back of the hemispherical analyzer. The camera image is captured and shown on computer screen using a program with a function to calculate the rotation angle

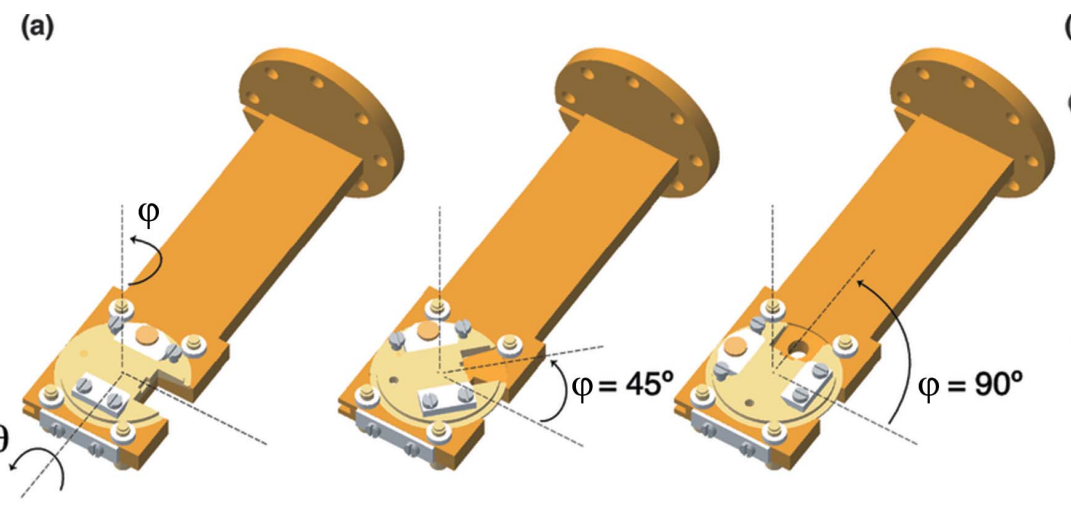

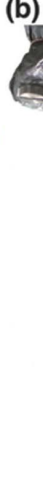

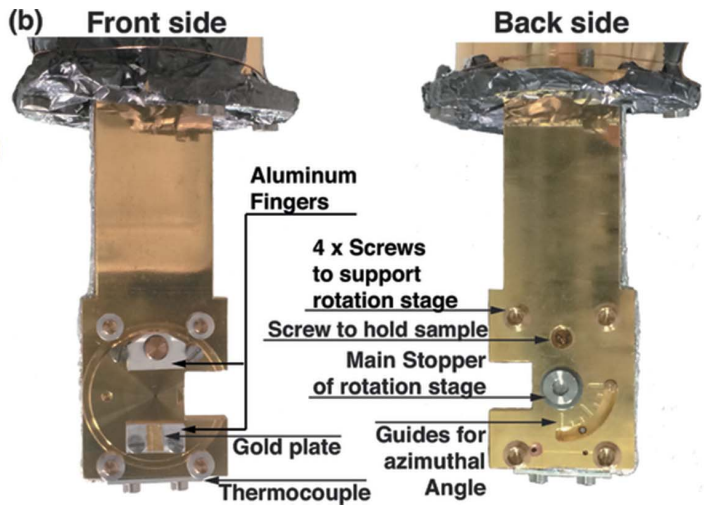

Figure 3

(a) Schematic view of our developed low-temperature two-axis manipulator with the definition of azimuthal angle $\varphi$ and polar angle $\theta$, and simulations for the azimuthal rotation with rotation angles of $45^{\circ}$ (middle) and $90^{\circ}$ (right). (b) Photographs of the manipulator taken from the front (left) and back (right). 
(a)

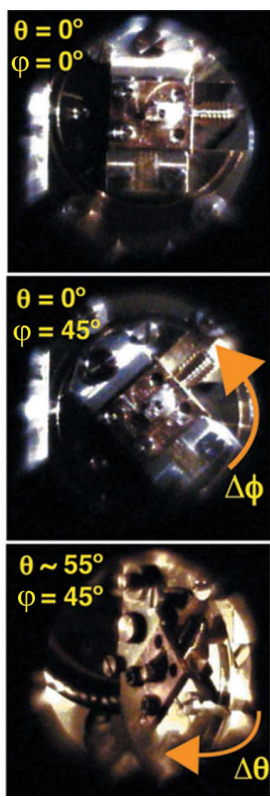

(b)

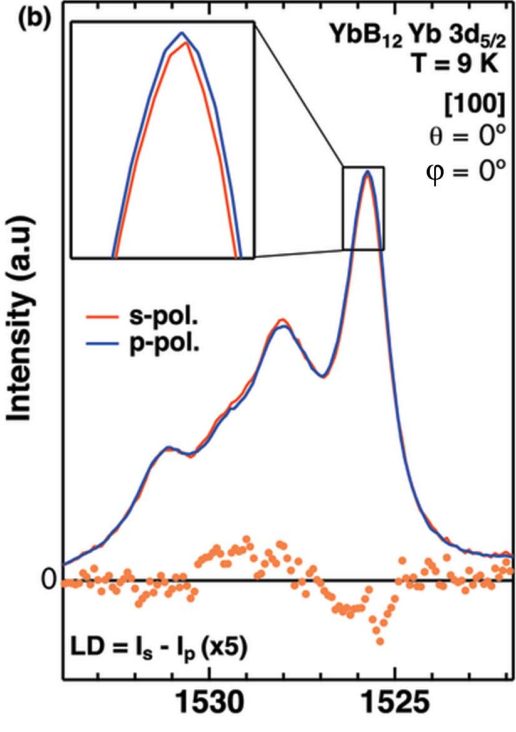

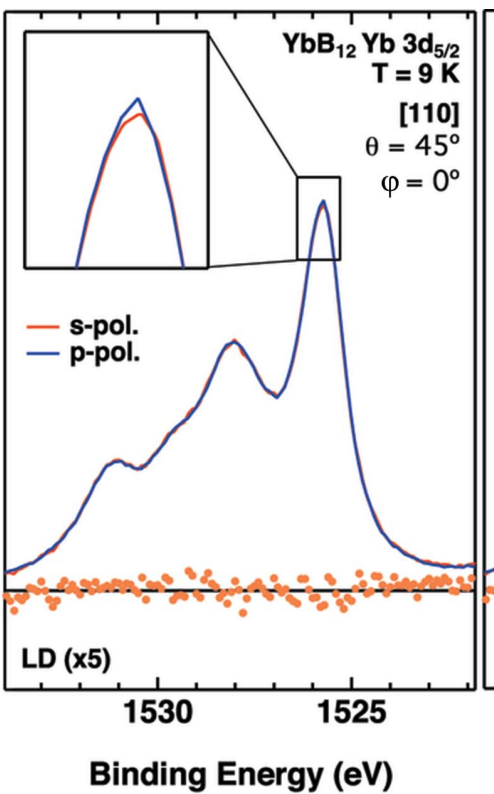

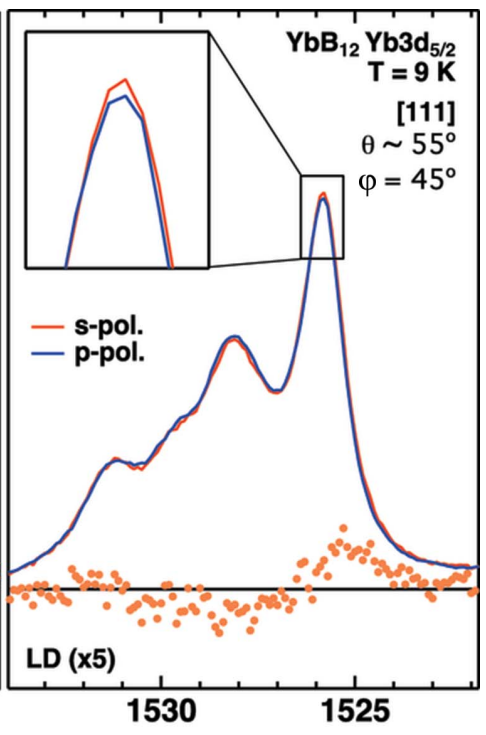

Figure 4

(a) CCD camera image of the manipulator (top) demonstrating the $45^{\circ}$ rotation for the azimuthal axis (middle) and further polar rotation of $\sim 55^{\circ}$ (bottom). (b) Linear polarization dependence of the $\mathrm{Yb}^{3+} 3 d_{5 / 2}$ core-level spectra for cubic $\mathrm{YbB}_{12}$ by selecting the photoelectron-detection directions [100] (left), [110] (middle) and [111] (right). Insets are close-ups of the $1525.5 \mathrm{eV}$ peak in the Yb ${ }^{3+} 3 d_{5 / 2}$ spectra. The so-called Shirly-type background is subtracted as discussed in the literature (Kanai et al., 2015).

with an accuracy of $\pm 1^{\circ}$. Fig. 4(a) shows the rotation procedures of the two-axis manipulator recorded by this CCD camera with magnification of $\sim 3$ achieved using a varifocal lens (Tamron: 13VM20100AS). The middle panel of Fig. 4(a) clearly shows the azimuthal rotation of $45^{\circ}$, and further polar rotation at $\sim 55^{\circ}$ (bottom).

By selecting the photoelectron-detection direction with the two-axis manipulator, we have studied the polarized HAXPES for a cubic strongly correlated rare-earth compound $\mathrm{YbB}_{12}$ (Kanai et al., 2015). Fig. 4(b) shows the $\mathrm{Yb}^{3+} 3 d_{5 / 2}$ corelevel spectra along the [100], [110] and [111] directions. Note that all three geometries have been set by the polar $\theta$ and azimuthal $\varphi$ rotations without any sample transfer procedure. In the [100] emission geometry for $(\theta, \varphi)=\left(0^{\circ}, 0^{\circ}\right)$, the $\mathrm{Yb}^{3+}$ $3 d$ core-level spectra show the polarization dependence especially at the peak at $1525.5 \mathrm{eV}$. As shown in the close-up of the peak top (inset), the $p$-polarized spectral weight is slightly stronger than the $s$-polarized spectral weight. This is highlighted by the linear dichroism (LD) in the core-level spectra (bottom) defined as $I_{\mathrm{s}}-I_{\mathrm{p}}$, where $I_{\mathrm{s}}\left(I_{\mathrm{p}}\right)$ denotes the intensity of $s$-polarized ( $p$-polarized) spectra. In the [100] emission geometry the LD spectrum changes sign near $1527 \mathrm{eV}$. This difference is noticeably suppressed in the [110] emission geometry by rotating the polar angle $\theta$ by $45^{\circ}$, and again shows up with the [111] emission geometry after rotation for both $\theta$ and $\varphi$ axis of the $(\theta, \varphi)=\left(\sim 55^{\circ}, 45^{\circ}\right)$ setting in Fig. 4(a). It is most interesting that the sign of LD for the [111] emission geometry is the reverse of that for the [100] emission, revealing the ground-state symmetry of the $\mathrm{Yb}^{3+} 4 f$ states due to the Coulomb and exchange interactions between the $\mathrm{Yb} 3 d$ core and $4 f$ holes (Mori et al., 2014; Kanai et al., 2015). Thus we stress that the selection of the photoelectron-detection direction is crucial for probing the ground-state symmetry for various strongly correlated electron systems.

\subsection{Co-axial monitoring system with long-working-distance microscope}

The two-axis rotation is very powerful for optimizing the detection direction of the photoelectrons, but it sometimes makes it difficult to normalize spectra recorded at different angles. This might be simply due to the surface roughness; the area of the excitation X-ray on a rough surface is often not exactly the same when the sample surface is not flat, yielding a variation of the photoemission intensity. In the case of a very weak dichroic signal, as shown in Fig. 4, it is crucial to record the photoemission signal from the same region on the sample surface for comparison of spectra before and after any rotations. To minimize this problem, we have also installed a coaxial sample monitoring system (Fig. 5) combining a longworking-distance optical microscope (Infinity photo-optical, $\mathrm{K} 2 / \mathrm{SC}$ ) and an aluminium mirror (50 $\mathrm{mm}$ square) having a $5 \mathrm{~mm}$-diameter through-hole for the X-ray beam as shown in Fig. 5(b).

As can be seen in Fig. 6(a), the sample position is selected using a micro-positioning technique with similar monitoring systems (Muro et al., 2009, 2011; Fujiwara et al., 2015). The intersection between the incoming X-ray beam and the photoelectron analyzer axis is marked beforehand onto the microscope monitor using a fluorescent substrate, which is positioned to maximize the counts of photoelectrons detected by the photoelectron analyzer. To adjust a certain region on the sample surface to this intersection, we first set the target region to the mark on the microscope monitor, and scanned 

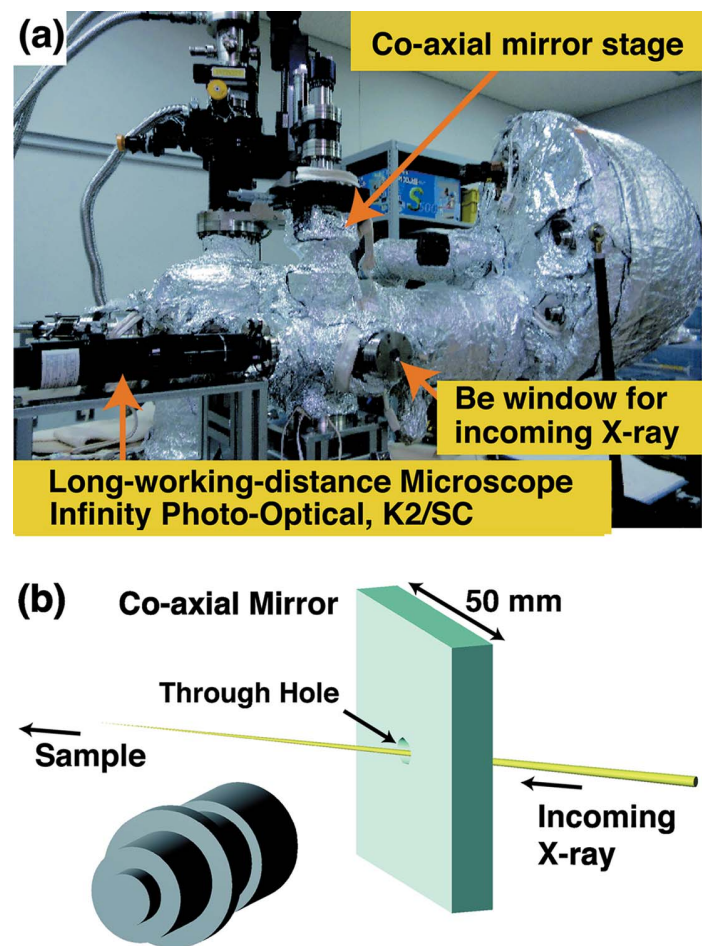

\section{Long-working-distance Microscope}

Figure 5

(a) Photograph of the co-axial sample monitoring system combining the long-working-distance microscope with co-axial mirror, and $(b)$ schematic view. The top view of the optical geometry is shown in Fig. 1.

the sample position along the beam axis while keeping the target region on the mark until detecting the maximum photoelectron-counts to avoid the ambiguity of the focal depth of the microscope. Note that we have a gold reference with a small amount of phosphor powder on the aluminium finger as shown in Fig. 3(b) to mark the beam spot any time as in Fig. 6(b). There is no serious problem caused by the phosphor powder on the gold surface for optimizing the count rate owing to the high photoelectron kinetic energy and long probing depth at HAXPES. This simple device is helpful if one changes the magnification of the microscope by changing the lens system of the microscope. The positioning error is within $\pm 50 \mu \mathrm{m}$ evaluated with the patterned Pt markers evaporated on $\mathrm{NiO}$ as shown in Figs. 6(c) and 6(d). This error is mainly due to vibration of the closed-cycle refrigerator.

By utilizing this positioning system we have studied the electronic structure of $\mathrm{NiO}$, which is a benchmark of strongly correlated insulators (Sawatzky \& Allen, 1984; Zaanen et al., 1985), for application as non-volatile resistive random access memory (Gibbons \& Beadle, 1964; Kim et al., 2006; Calka et al., 2011; Jeong et al., 2012; Horiba et al., 2013). We applied a voltage to the $\mathrm{NiO}$ film by the Pt point contact for switching the resistivity at 50 places in a region of $200 \mu \mathrm{m} \times 200 \mu \mathrm{m}$, indicated by the square-shaped $\mathrm{Pt}$ marker on the $\mathrm{NiO}$ as shown in Figs. 6(c) and 6(d). We set the metallic states on $\mathrm{NiO}$ by sweeping the voltage from $0 \mathrm{~V}$ to $+20 \mathrm{~V}$ between the $\mathrm{Pt}$ point contact touching on the $\mathrm{NiO}$ film and the p-type $\mathrm{Si}$ substrate, as shown in Fig. $6(e)$, then release the voltage to $0 \mathrm{~V}$
Fractured sample surface

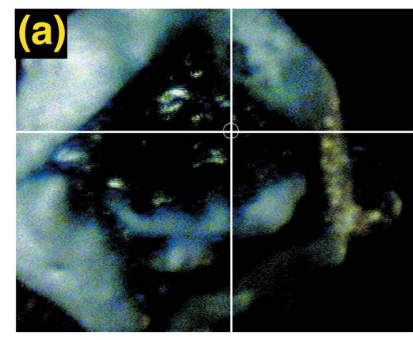

Fluorescence powder reference

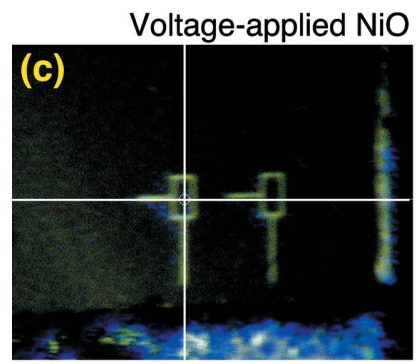

(e)
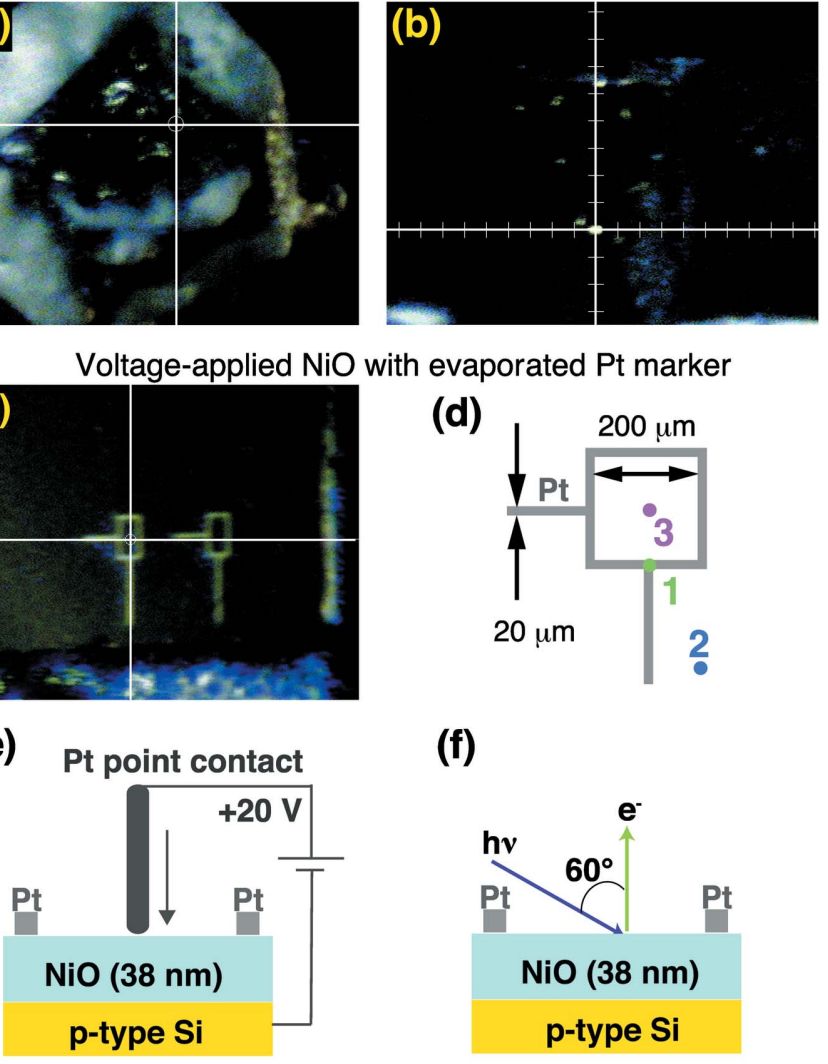

evaporated Pt marker

(d)

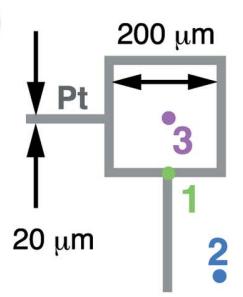

(f)

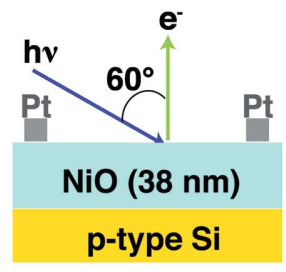

Figure 6

Co-axial microscope image of the fractured surface of $\mathrm{YbB}_{12}(a)$, of the fluorescence-powder reference on the gold reference on the manipulator (b), and of the evaporated Pt marker on NiO film $(c)$. The X-ray beam spot is located at the intersection of the cross-lines. (d) Schematic image of the square- and line-shaped Pt marker for defining specific regions on the NiO. The positions measured by HAXPES are also indicated on the $\mathrm{Pt}$ marker (1), as-grown $\mathrm{NiO}$ film (2) and the voltage-applied region on $\mathrm{NiO}$ (3). (e) Schematic view of the setup for applying the voltage on the $\mathrm{NiO}$ film by $\mathrm{Pt}$ point contact outside the photoemission chamber, and $(f)$ the experimental geometry of the HAXPES measurement.

while keeping the metallic conductivity due to the non-volatility. For studying the electronic structures of this sample by HAXPES in a different chamber using synchrotron radiation, we have chosen three measurement positions: the Pt marker (position 1), the as-grown $\mathrm{NiO}$ film (position 2) and the voltage-applied $\mathrm{NiO}$ (position 3), indicated in Fig. 6(d). The closed-cycle He refrigerator was switched off to minimize the vibration of the manipulator with the positioning error to $\pm 25 \mu \mathrm{m}$. Thus the measured temperature was set to $300 \mathrm{~K}$ and the overall energy resolution was set to $400 \mathrm{meV}$ with the normal emission geometry as shown in Fig. $6(f)$.

Fig. 7 shows polarization-dependent valence-band HAXPES spectra recorded at these three positions. The valence-band spectra recorded on the Pt marker, namely at position 1, show the clear Fermi cut-off, and the spectral shape is not very sensitive to the polarization (Yamasaki et al., 2014). Meanwhile, the spectra for as-grown $\mathrm{NiO}$ at position 2 outside the Pt marker clearly show the gap opening near the Fermi level. Here clear polarization dependence of the line shape is observed; namely, the $\mathrm{Ni} 4 s$ contribution is much enhanced in 


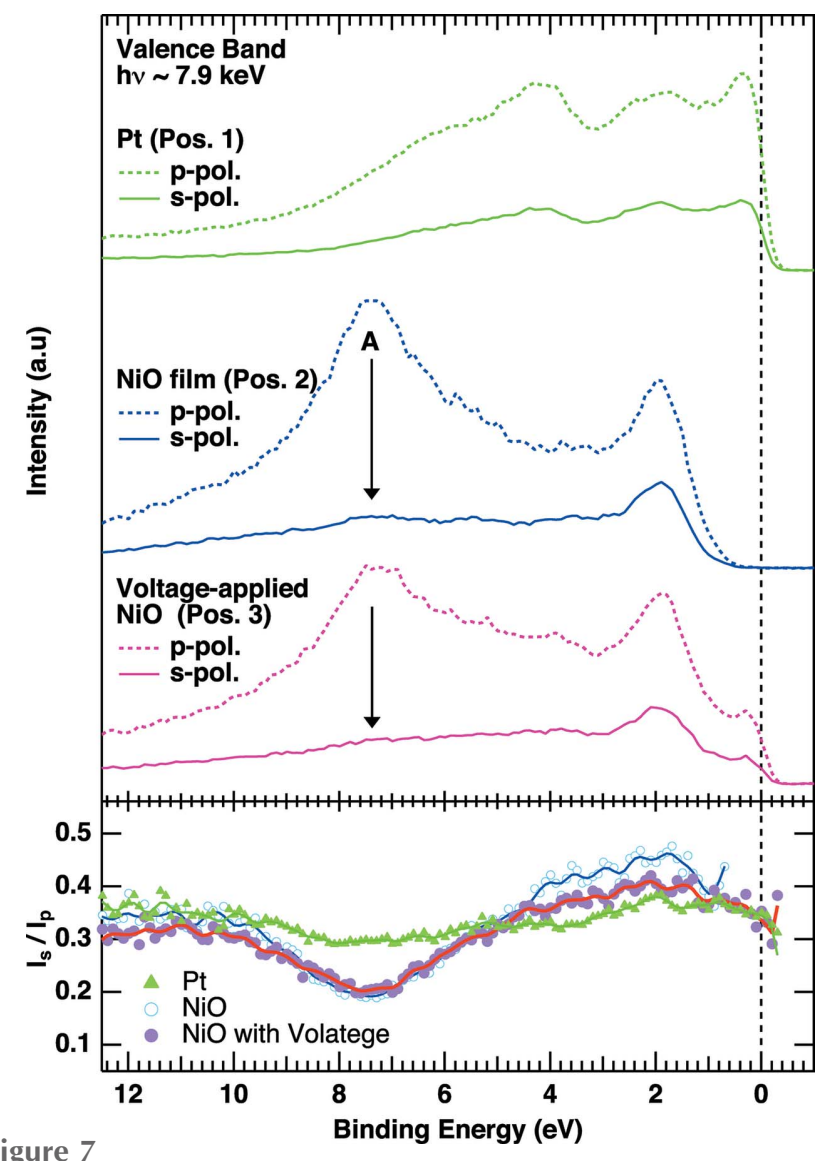

Valence-band photoemission spectra of Pt measured at position 1 (Pos. 1) in Fig. 6(d), those of the as-grown NiO substrate (Pos. 2) and those of the voltage-applied $\mathrm{NiO}$ (Pos. 3) (bottom) recorded by the $p$ - and $s$-polarized photons. $I_{\mathrm{s}} / I_{\mathrm{p}}$ spectra for all three positions are plotted in the lower panel. Note that the data points ranging from $0.4 \mathrm{eV}$ to the Fermi level in the $I_{\mathrm{s}} / I_{\mathrm{p}}$ spectrum for as-grown $\mathrm{NiO}$ are removed to eliminate the divergent behavior due to the gap opening.

the $p$-polarized geometry, especially at the position of peak A around $7.5 \mathrm{eV}$ (Sekiyama et al., 2010; Nakatsu et al., 2011; Weinen et al., 2015). This is due to the asymmetry factors of the photoionization cross sections (Trzhaskovskaya et al., 2001, 2002, 2006). We note that the spectral line shape of the valence-band spectra of the as-grown $\mathrm{NiO}$ is essentially the same as that reported by Weinen et al. (2015), in which the valence-band spectra of $\mathrm{NiO}$ were recorded in the complete $s$ and $p$-polarized geometries by changing the analyzer position. Therefore we stress that our polarization switching system works successfully with a high degree of linear polarization on the sample position.

Compared with the as-grown $\mathrm{NiO}$, the metallic Fermi cutoff is clearly observed at position 3 in the voltage-applied $\mathrm{NiO}$ in Fig. $6(d)$. To identify the origin of the metallic cut-off, we have compared the intensity ratio of the $s$-polarized ( $p$ polarized) spectra $\left(I_{\mathrm{s}} / I_{\mathrm{p}}\right)$ for all three positions in the lower panel of Fig. 7, for resolving the orbital contributions in the valence-band spectra (Sekiyama et al., 2010). $I_{\mathrm{s}} / I_{\mathrm{p}}$ for the voltage-applied $\mathrm{NiO}$ overlaps the as-grown spectra in the range from $12 \mathrm{eV}$ to $5 \mathrm{eV}$, suggesting the valence-band character is not heavily modified in this binding energy range after application of the voltage. Meanwhile, $I_{\mathrm{s}} / I_{\mathrm{p}}$ for the voltageapplied $\mathrm{NiO}$ ranging from $5 \mathrm{eV}$ to $1 \mathrm{eV}$ is somewhat in between those of the $\mathrm{Pt}$ and as-grown $\mathrm{NiO}$, and overlaps fully with that of Pt near the Fermi level, suggesting a contribution from $\mathrm{Pt}$ in this region on the voltage-applied $\mathrm{NiO}$. We have further compared the spectral line shape of voltage-applied $\mathrm{NiO}$ with the $\mathrm{Pt}$ valence-band spectra in Fig. 8(a). The normalized Pt valence-band spectrum, so as to fit the slope of the metallic Fermi cut-off, completely overlaps with the spectrum of the voltage-applied NiO near the Fermi level. The subtracted spectrum of the normalized Pt spectrum from the raw spectrum of the voltage-applied $\mathrm{NiO}$ quantitatively reproduces the spectrum of the as-grown NiO. The $\mathrm{Pt}$ contribution in the voltage-applied $\mathrm{NiO}$ is also supported by a weak but finite spectral weight due to the $\mathrm{Pt} 4 p_{3 / 2}$ core-level contributions in Fig. 8(b). We should note that the Pt contribution is not due to the surrounded $\mathrm{Pt}$ because the length of
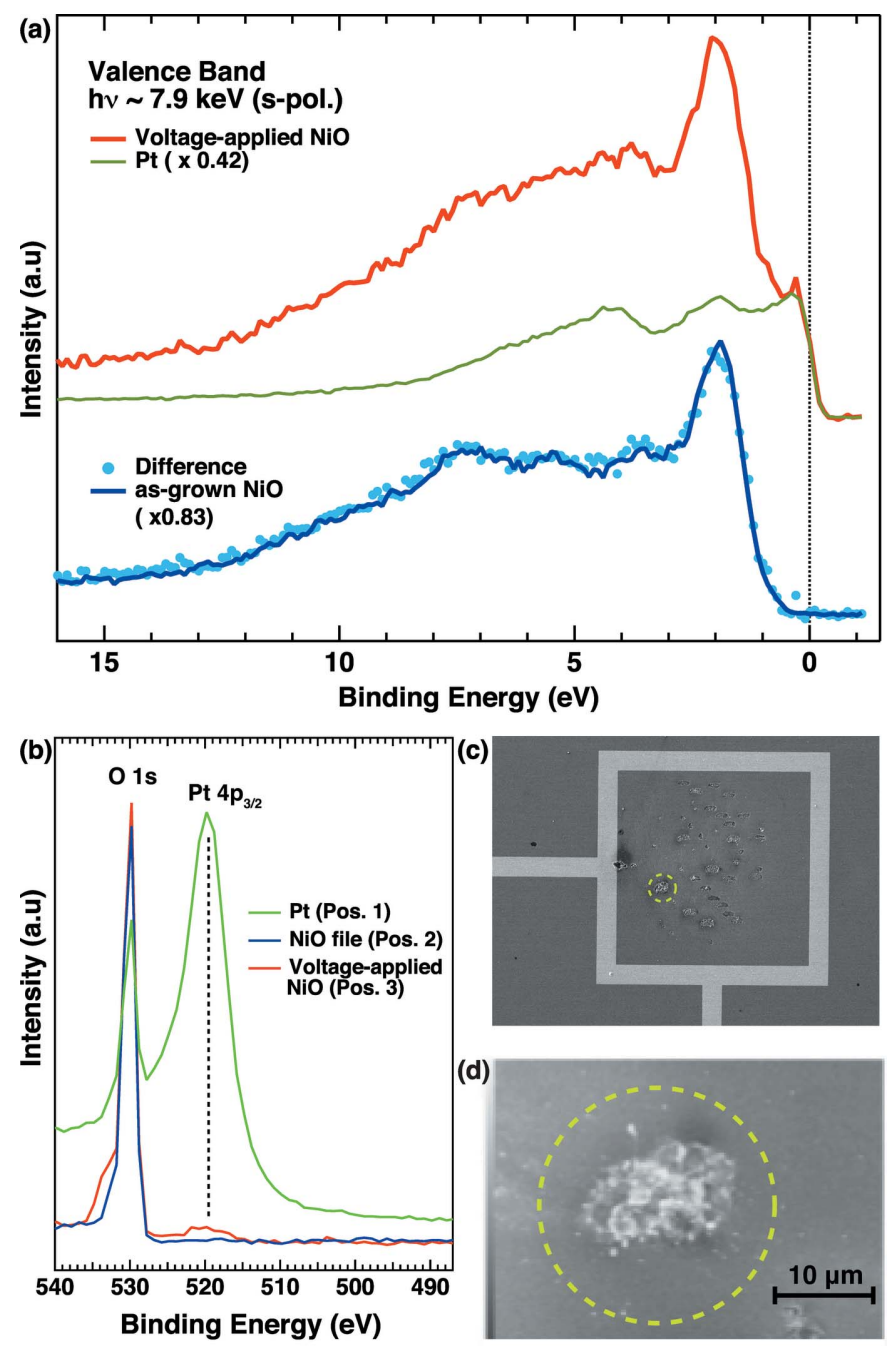

Figure 8

(a) Valence-band photoemission spectrum with $s$-polarization geometry for the voltage-applied $\mathrm{NiO}$ and normalized spectrum of $\mathrm{Pt}$ (top). The difference is shown at the bottom with the spectrum of the as-grown $\mathrm{NiO}$ film. (b) Pt $4 p_{3 / 2}$ core-level photoemission spectra. (c) SEM image for the voltage-applied $\mathrm{NiO}$. The dashed circle indicates one of the spots touched by the Pt point contact, with a focused SEM image shown in $(d)$. 
the inner side of the marker is $200 \mu \mathrm{m}$ while the footprint of the X-ray beam on the sample surface is $25 \mu \mathrm{m} \times 50 \mu \mathrm{m}$ (FWHM) for the normal emission geometry with grazing angle of $30^{\circ}$ in which the sample normal is inclined by $60^{\circ}$ to the X-ray beam as shown in Fig. $6(f)$. Since the spatial distribution of the photoelectrons recorded by the photoelectron spectrometer was well focused, the signal from the surrounded Pt can be excluded from the spectral shape of the voltage-applied $\mathrm{NiO}$.

Indeed, the scanning tunneling microscope (SEM) image for the region of voltage-applied $\mathrm{NiO}$ shows many imprints formed by applying the voltage with Pt point contact as shown in Fig. 8(c). In particular, the focused SEM image (Fig. $8 d$ ) for one of the imprints indicated by the dashed circle in Fig. 8(c) clearly shows a bump-like structure, where the energydispersive X-ray image (not shown) revealed the fluorescence signals from the Pt $M$-edge. Therefore, the origin of the metallic cut-off could be due to diffused $\mathrm{Pt}$ from the point contact during application of the voltages for switching the resistivity. This provides important feedback for improving the method of preparation of the electrical contact for studying the electronic structure of NiO-based resistive random access memory.

\section{Conclusion}

We have developed a HAXPES system equipped with a highthroughput X-ray phase retarder, a low-temperature two-axis manipulator and a co-axial microscope for revealing the ground-state symmetry of strongly correlated electron systems. The two-axis manipulator is capable of rotating by $90^{\circ}$ for azimuthal and polar angles with a lowest cooling temperature achieved of $9 \mathrm{~K}$ by closed-cycle He refrigerator, which gives a better chance to study the electronic structure of the ground states in various rare-earth compounds. The coaxial sample monitoring system with a long-working-distance optical microscope supports recording of the reliable polarized HAXPES spectra by keeping the same sample position before and after rotation. By utilizing this micro-positioning system, we have demonstrated linear polarization-dependent valence-band HAXPES for voltage-applied $\mathrm{NiO}$ in order to study the resistive switching phenomena and found that $\mathrm{Pt}$ could be diffused on $\mathrm{NiO}$ when a voltage was applied, forming metallic states near the Fermi level.

\section{Acknowledgements}

This work was supported by a Grant-in-Aid for Scientific Research (23654121), Grants-in-Aid for Young Scientists (23684027, 23740240) and a Grant-in-Aid for Innovative Areas (20102003) from MEXT and JSPS, Japan, and by Toray Science Foundation. Part of the NiO sample preparation was supported by Nihon University College of Science and Technology Grants-in-Aid for Fundamental Science Research (2014). We are grateful to F. Iga for providing $\mathrm{YbB}_{12}$ samples. HF thanks K. Yano, S. Fujioka, H. Aratani, Y. Nakatani and $\mathrm{K}$. Terashima for supporting the experiment. The hard X-ray photoemission was performed at SPring-8 under the approval of JASRI (2014A1149, 2014B1305).

\section{References}

Calka, P., Martinez, E., Lafond, D., Minoret, S., Tirano, S., Detlefs, B., Roy, J., Zegenhagen, J. \& Guedj, C. (2011). J. Appl. Phys. 109, 124507.

Fujiwara, H., Kiss, T., Wakabayashi, Y. K., Nishitani, Y., Mori, T., Nakata, Y., Kitayama, S., Fukushima, K., Ikeda, S., Fuchimoto, H., Minowa, Y., Mo, S.-K., Denlinger, J. D., Allen, J. W., Metcalf, P., Imai, M., Yoshimura, K., Suga, S., Muro, T. \& Sekiyama, A. (2015). J. Synchrotron Rad. 22, 776-780.

Gibbons, J. F. \& Beadle, W. E. (1964). Solid State Electron. 7, 785790.

Giles, C., Malgrange, C., Goulon, J., de Bergevin, F., Vettier, C., Dartyge, E., Fontaine, A., Giorgetti, C. \& Pizzini, S. (1994). J. Appl. Cryst. 27, 232-240.

Hansmann, P., Severing, A., Hu, Z., Haverkort, M. W., Chang, C. F., Klein, S., Tanaka, A., Hsieh, H. H., Lin, H.-J., Chen, C. T., Fåk, B., Lejay, P. \& Tjeng, L. H. (2008). Phys. Rev. Lett. 100, 066405.

Hirano, K., Ishikawa, T. \& Kikuta, S. (1993). Nucl. Instrum. Methods Phys. Res. A, 336, 343-353.

Hirano, K., Izumi, K., Ishikawa, T., Annaka, S. \& Kikuta, S. (1991). Jpn. J. Appl. Phys. 30, L407-L410.

Hirano, K. \& Maruyama, H. (1997). Jpn. J. Appl. Phys. 36, L1272L1274.

Horiba, K., Fujiwara, K., Nagamura, N., Toyoda, S., Kumigashira, H., Oshima, M. \& Takagi, H. (2013). Appl. Phys. Lett. 103, 193114.

Jeong, D. S., Thomas, R., Katiyar, R. S., Scott, J. F., Kohlstedt, H., Petraru, A. \& Hwang, C. S. (2012). Rep. Prog. Phys. 75, 076502.

Kanai, Y., Mori, T., Naimen, S., Yamagami, K., Fujiwara, H., Higashiya, A., Kadono, T., Imada, S., Kiss, T., Tanaka, A., Tamasaku, K., Yabashi, M., Ishikawa, T., Iga, F. \& Sekiyama, A. (2015). J. Phys. Soc. Jpn, 84, 073705.

Kim, D. C., Seo, S., Ahn, S. E., Suh, D.-S., Lee, M. J., Park, B.-H., Yoo, I. K., Baek, I. G., Kim, H.-J., Yim, E. K., Lee, J. E., Park, S. O., Kim, H. S., Chung, U. I., Moon, J. T. \& Ryu, B. I. (2006). Appl. Phys. Lett. 88, 202102-1-3.

Kitamura, H., Bizen, T., Hara, T., Maréchal, X. M., Seike, T. \& Tanaka, T. (2001). Nucl. Instrum. Methods Phys. Res. A, 467-468, 110-113.

Kozina, X., Fecher, G. H., Stryganyuk, G., Ouardi, S., Balke, B., Felser, C., Schönhense, G., Ikenaga, E., Sugiyama, T., Kawamura, N., Suzuki, M., Taira, T., Uemura, T., Yamamoto, M., Sukegawa, H., Wang, W., Inomata, K. \& Kobayashi, K. (2011). Phys. Rev. B, 84, 054449.

Mori, T., Kitayama, S., Kanai, Y., Naimen, S., Fujiwara, H., Higashiya, A., Tamasaku, K., Tanaka, A., Terashima, K., Imada, S., Yasui, A., Saitoh, Y., Yamagami, K., Yano, K., Matsumoto, T., Kiss, T., Yabashi, M., Ishikawa, T., Suga, S., Ōnuki, Y., Ebihara, T. \& Sekiyama, A. (2014). J. Phys. Soc. Jpn, 83, 123702.

Muro, T., Kato, Y., Matsushita, T., Kinoshita, T., Watanabe, Y., Okazaki, H., Yokoya, T., Sekiyama, A. \& Suga, S. (2011). J. Synchrotron Rad. 18, 879-884.

Muro, T., Kato, Y., Matsushita, T., Kinoshita, T., Watanabe, Y., Sekiyama, A., Sugiyama, H., Kimura, M., Komori, S., Suga, S., Okazaki, H. \& Yokoya, T. (2009). Rev. Sci. Instrum. 80, 053901.

Nakatsu, Y., Sekiyama, A., Imada, S., Okamoto, Y., Niitaka, S., Takagi, H., Higashiya, A., Yabashi, M., Tamasaku, K., Ishikawa, T. \& Suga, S. (2011). Phys. Rev. B, 83, 115120.

Okitsu, K., Ueji, Y., Sato, K. \& Amemiya, Y. (2001). J. Synchrotron Rad. 8, 33-37.

Ouardi, S., Fecher, G. H., Kozina, X., Stryganyuk, G., Balke, B., Felser, C., Ikenaga, E., Sugiyama, T., Kawamura, N., Suzuki, M. \& Kobayashi, K. (2011). Phys. Rev. Lett. 107, 036402.

Sawatzky, G. A. \& Allen, J. W. (1984). Phys. Rev. Lett. 53, 2339-2342. 
Scagnoli, V., Mazzoli, C., Detlefs, C., Bernard, P., Fondacaro, A., Paolasini, L., Fabrizi, F. \& de Bergevin, F. (2009). J. Synchrotron Rad. 16, 778-787.

Sekiyama, A., Higashiya, A. \& Imada, S. (2013). J. Electron Spectrosc. Relat. Phenom. 190, 201-204.

Sekiyama, A., Yamaguchi, J., Higashiya, A., Obara, M., Sugiyama, H., Kimura, M. Y., Suga, S., Imada, S., Nekrasov, I. A., Yabashi, M., Tamasaku, K. \& Ishikawa, T. (2010). New J. Phys. 12, 043045.

Strigari, F., Willers, T., Muro, Y., Yutani, K., Takabatake, T., Hu, Z., Agrestini, S., Kuo, C.-Y., Chin, Y.-Y., Lin, H.-J., Pi, T. W., Chen, C. T., Weschke, E., Schierle, E., Tanaka, A., Haverkort, M. W., Tjeng, L. H. \& Severing, A. (2013). Phys. Rev. B, 87, 125119.

Strigari, F., Willers, T., Muro, Y., Yutani, K., Takabatake, T., Hu, Z., Chin, Y.-Y., Agrestini, S., Lin, H.-J., Chen, C. T., Tanaka, A., Haverkort, M. W., Tjeng, L. H. \& Severing, A. (2012). Phys. Rev. B, 86, 081105 .

Trzhaskovskaya, M. B., Nefedov, V. I. \& Yarzhemsky, V. G. (2001). At. Data Nucl. Data Tables, 77, 97-159.

Trzhaskovskaya, M. B., Nefedov, V. I. \& Yarzhemsky, V. G. (2002). At. Data Nucl. Data Tables, 82, 257-311.

Trzhaskovskaya, M. B., Nikulin, V. K., Nefedov, V. I. \& Yarzhemsky, V. G. (2006). At. Data Nucl. Data Tables, 92, 245-304.

Ueda, S., Tanaka, H., Takaobushi, J., Ikenaga, E., Kim, J.-J., Kobata, M., Kawai, T., Osawa, H., Kawamura, N., Suzuki, M. \& Kobayashi, K. (2008). Appl. Phys. Express, 1, 077003.

Weinen, J., Koethe, T. C., Chang, C. F., Agrestini, S., Kasinathan, D., Liao, Y. F., Fujiwara, H., SchüSSSler-Langeheine, C., Strigari, F., Haupricht, T., Panaccione, G., Offi, F., Monaco, G., Huotari, S., Tsuei, K.-D. \& Tjeng, L. H. (2015). J. Electron Spectrosc. Relat. Phenom. 198, 6-11.
Willers, T., Adroja, D. T., Rainford, B. D., Hu, Z., Hollmann, N., Körner, P. O., Chin, Y.-Y., Schmitz, D., Hsieh, H. H., Lin, H.-J., Chen, C. T., Bauer, E. D., Sarrao, J. L., McClellan, K. J., Byler, D., Geibel, C., Steglich, F., Aoki, H., Lejay, P., Tanaka, A., Tjeng, L. H. \& Severing, A. (2012). Phys. Rev. B, 85, 035117.

Willers, T., Fåk, B., Hollmann, N., Körner, P. O., Hu, Z., Tanaka, A., Schmitz, D., Enderle, M., Lapertot, G., Tjeng, L. H. \& Severing, A. (2009). Phys. Rev. B, 80, 115106.

Willers, T., Hu, Z., Hollmann, N., Körner, P. O., Gegner, J., Burnus, T., Fujiwara, H., Tanaka, A., Schmitz, D., Hsieh, H. H., Lin, H.-J., Chen, C. T., Bauer, E. D., Sarrao, J. L., Goremychkin, E., Koza, M., Tjeng, L. H. \& Severing, A. (2010). Phys. Rev. B, 81, 195114.

Willers, T., Strigari, F., Hu, Z., Sessi, V., Brookes, N. B., Bauer, E. D., Sarrao, J. L., Thompson, J. D., Tanaka, A., Wirth, S., Tjeng, L. H. \& Severing, A. (2015). Proc. Natl Acad. Sci. 112, 23842388.

Yabashi, M., Mochizuki, T., Yamazaki, H., Goto, S., Ohashi, H., Takeshita, K., Ohata, T., Matsushita, T., Tamasaku, K., Tanaka, Y. \& Ishikawa, T. (2001a). Nucl. Instrum. Methods Phys. Res. A, 467468, 678-681.

Yabashi, M., Tamasaku, K. \& Ishikawa, T. (2001b). Phys. Rev. Lett. 87, 140801-1-4.

Yamasaki, A., Tachibana, S., Fujiwara, H., Higashiya, A., Irizawa, A., Kirilmaz, O., Pfaff, F., Scheiderer, P., Gabel, J., Sing, M., Muro, T., Yabashi, M., Tamasaku, K., Sato, H., Namatame, H., Taniguchi, M., Hloskovskyy, A., Yoshida, H., Okabe, H., Isobe, M., Akimitsu, J., Drube, W., Claessen, R., Ishikawa, T., Imada, S., Sekiyama, A. \& Suga, S. (2014). Phys. Rev. B, 89, 121111.

Zaanen, J., Sawatzky, G. A. \& Allen, J. W. (1985). Phys. Rev. Lett. 55, 418-421. 